\title{
ROBOTIC VIBRO ENGINE MOTION ANALYSIS
}

Edgars Kovals, Kaspars Burbeckis, Kristaps Spade, Janis Viba

Riga Technical University, Latvia

edgars.kovals@gmail.com, kaspars.burbeckis@gmail.com, kristaps.spade@rtu.lv, janis.viba@rtu.lv

\begin{abstract}
Vibro engine movement is viewed in a horizontal or inclined plane. The mathematical model includes a complete body of motion with a sliding or rotating engine inside (with extra mass). In general, the system has a minimum of four degrees of freedom (three degrees of freedom for the frame, one for relative motion of the internal mass). If, in addition to the vibro engine (robot), the towed object is added, then there may even be five to seven degrees of freedom. In the process of generating system motion, additional micro-compression with the foundation takes place. Computer technology was used in the study of the dynamics of such a complex object. It has been found that the main parameter of the drag effect is the slip friction coefficient, which should be chosen as maximally possible or as necessary to use sharps. The results of modeling are presented. For example, it is noted that the average speed can be up to $0.5 \mathrm{~m} \cdot \mathrm{s}^{-1}$. The ability to pull a load on a robot has been analyzed. It is understood that in this case the driving speed is reduced. The system can be used for moving robotic objects.
\end{abstract}

Keywords: bodies collisions, oblique impact, vibrotransport, robotic driver.

\section{Introduction}

At one point in the solid body collision process, the analytical solution is only possible in the case of complete motion [1-4]. However, there are also many (up to seven) cases of collision in this case, too [5]. To find them, you need to know the starting rules of the movement, the body configurations and the material for making it. The series-type collision process must use gradual calculations at the same point of the previous collision, changing the starting conditions. The question of how to analyze concurrent collisions at several points analytically is not explored [6]. Theoretically, several collisions between multiple body systems at several points have been not studied. In all these cases, there are the following problems: one-sided impact interaction in the normal direction; stop the interaction in the equilibrium area; the tangential direction of the points of contact must be respected [5]. Despite these difficulties, the engineers invent and implement various technological processes with collisions of several large and small objects. For example, collision in vibrotransport, vibro separation, vibro polishing is widely used (Fig. 1.) [7]. Computer software is used to analyze such complex processes in today's technic and technology. In this article, we will look at the possibilities of using MathCAD and Working Model programs [8].

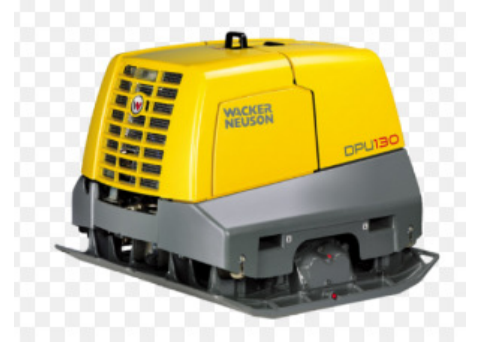

Fig. 1. Vibratory plate compactor [3]

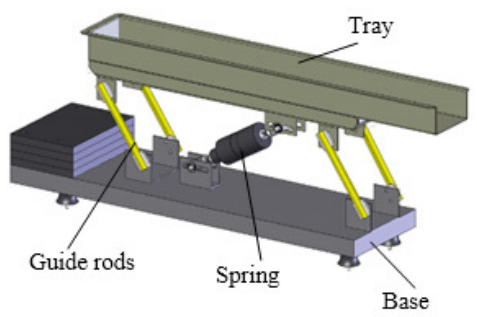

Fig. 2. Vibrating conveyors [4]

\section{Analysis of plane collisions in one or two points}

Plane collision rigid body against obstacle may be investigated by classical mechanics laws, using the momentum exchange theorem before and after collision in infinitive small time interval $[5 ; 6]$. In the synthesis of vibrating engines, let us analyze the body's collisions right and left at the contact points A and B (Fig. 3.). Also, we will analyze the simultaneous collision at both contact points A, B. Here we only look at the most difficult two-point collision (Fig.3, case 3 - collisions in two points). Let us determine the parameters at which the transport movement starts. We will also find jumping conditions.

The calculation is given by equation (1-10) using: momentum exchange theorem (1-5), for a rigid body with mass $m$; moment of momentum exchange theorem against the center mass of body with 
moment inertia $J C$ against centre mass (3), (6); normal impulsis restitution coefficent $R(7),(8)$ and the dry friction coefficient $f(9),(10)$. Case of starting rotation with the angular velocity $\omega 1$ about fixed point $A$ gives equations system (1-6). It is assumed that the body springs back by the angular velocity $\omega 2$. The calculation uses the resting state of body in the middle of the impact, which is estimated in the equations with a value of zero for the center of gravity velocity and rotational velocity. Letter $A$ means that the forces normal $\mathrm{N}$ and tangential $\mathrm{F}$ directions concern to the left point $A$. The letter $B$ concerns the right point $B$. Letter $S$ means that it is an impulse of a given force. Other parameters are shown in Fig. 3.

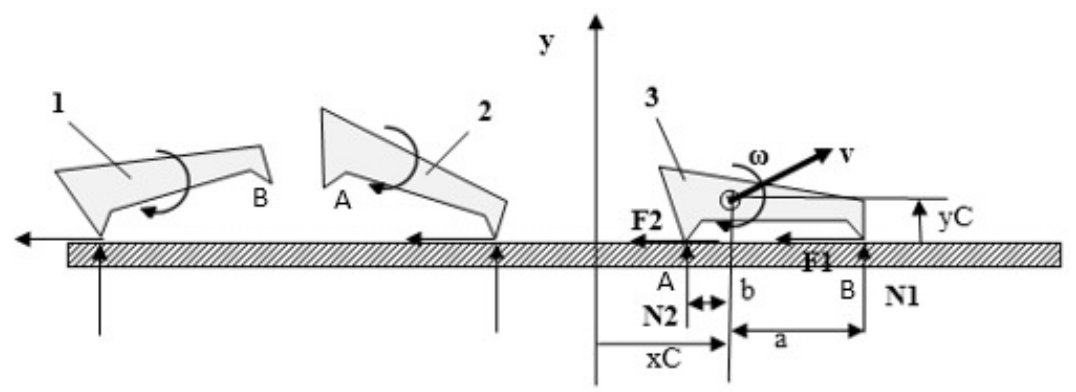

Fig. 3. Collision cases: 1 - left collision; 2 - right collision; 3 - collisions in two points A and B

In equations (7), (8), it is assumed that the coefficient of impulses restitution $R$ is the same in both contact points. Additionally, it is assumed that the collision process is on the limit of sliding in the left point A. It allows calculating the minimal friction coefficient $f$ from equation (11). If the real friction coefficient is less $f$, transport movement starts and this case can be used for the robotic vibro engine. Additionally, normal impulse SNA1 from formula (12) can be calculated. If it is negative, spring motion starts and three degree of freedom (3DOF) motion analysis can be used with step by step impact right and left sides. For that case and more than one rigid bodies collision and transport motion, computer discret model programs can be used. This is further demonstrated in our work on several robot engine models. As a result, the examples will be shown in a given main objective - how to use MathCAD and Working Model program of robotic exploration and vibro engine design synthesis.

$$
\begin{gathered}
0-m \cdot \omega 1 \cdot y C=S F A 1-S F B 1 ; \\
0-(-m \cdot \omega 1 \cdot x C)=S N A 1+S N B 1 ; \\
0-\left\{-\left[J C+m \cdot\left(x C^{2}+y C^{2}\right)\right] \cdot \omega 1\right\}=(a+b) \cdot S N B 1 ; \\
-m \cdot \omega 2 \cdot y C-0=S F A 2-S F B 2 ; \\
m \cdot \omega 2 \cdot x C-0=S N A 2+S N B 2 ; \\
{\left[J C+m \cdot\left(x C^{2}+y C^{2}\right)\right] \cdot \omega 2-0=(a+b) \cdot S N B 2 ;} \\
S N A 2=R \cdot S N A 1 ; \\
S N B 2=R \cdot S N B 1 ; \\
S F B 1=0 ; \\
S F A 1=f \cdot S N A 1, \\
m \cdot y C \cdot(a+b) \\
f=\frac{m A 1=\frac{\omega 1 \cdot\left\{m \cdot x C \cdot(a+b)-\left[J C+m \cdot\left(x C^{2}+y C^{2}\right)\right]\right\}}{a+b} .}{J C+m \cdot\left(x C^{2}+y C^{2}\right)-m \cdot x C \cdot(a+b)} ;
\end{gathered}
$$




\section{Analysis of vibrodrivers of one degree of freedom (1DOF) motion of two mass system by MathCAD}

The horizontal model of the system is shown in Fig. 4. With the given model it is possible to demonstrate the movement of the object's vibrotransport. Assuming that the actuator provides the rotation movement of the selected rods at an angle $\beta$, the differential equation of motion for mass $\mathrm{m} 1$ is as follows:

$$
\begin{aligned}
& (m 1+m 2) \ddot{x} 1=m 2\left\{L \left[(v \beta)^{2} \sin (\beta)+\right.\right. \\
& +a \beta \cdot \cos (\beta)]-f\{(m 1+m 2) g+ \\
& \left.+m 2 L\left[-(v \beta)^{2} \cos (\beta)-a \beta \cdot \sin (\beta)\right]\right\} \operatorname{sign}(\dot{x} 1),
\end{aligned}
$$

where $L$ - length of rods;

$f$ - coefficient of friction;

$\ddot{x} 1, \dot{x} 1-$ acceleration and velocity of mass 1 ;

$v \beta, a \beta-$ first and second derivation of angle $\beta$;

$g-$ free fall acceleration.

Example of motion modeling, accordingly to control action in Fig. 5 is shown in Fig. 5-8. Middle velocity is about $0.5 \mathrm{~m} \cdot \mathrm{s}^{-1}$.

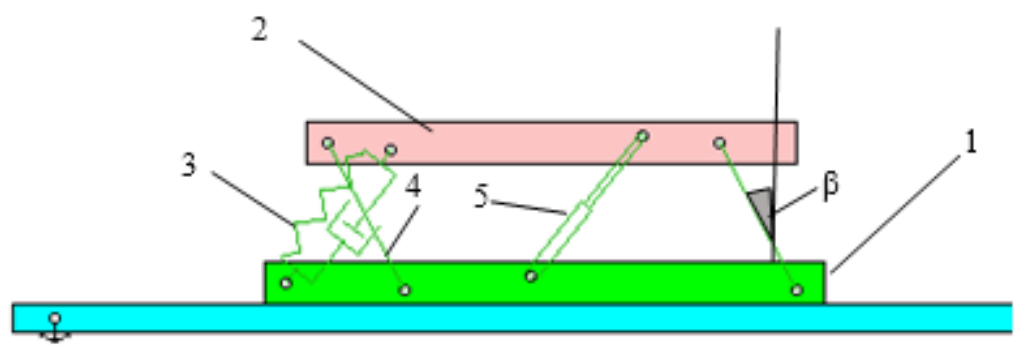

Fig. 4. Horizontal vibrodriver: 1 - base mass $\mathrm{m} 1 ; 2$ - upper mass $\mathrm{m} 2$;

3 - spring with dumper; 4 - rods; 5 - actuator

$$
\begin{aligned}
& \beta_{n}:=\beta 0+A \cdot \sin \left(\omega \cdot t_{n}\right) \\
& v \beta_{n}:=0+A \cdot \omega \cdot \cos \left(\omega \cdot t_{n}\right) \\
& a \beta_{n}=-\omega^{2} \cdot A \cdot \sin \left(\omega \cdot t_{n}\right)
\end{aligned}
$$

Fig. 5. Programm control of angle $\beta$

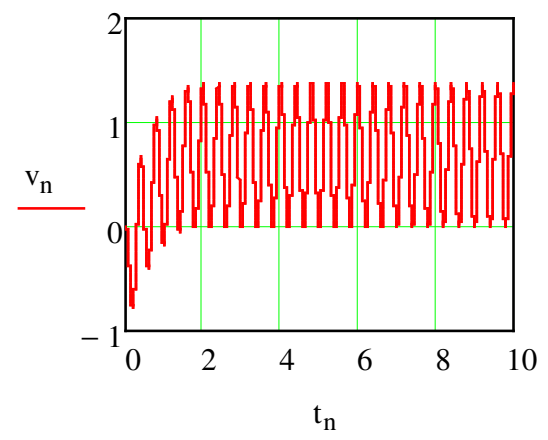

Fig. 7. Velocity graphics

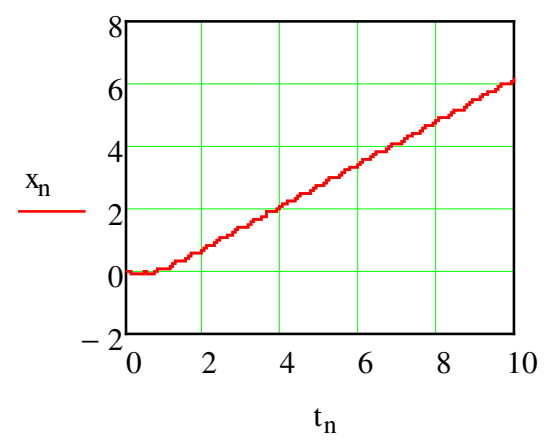

Fig. 6. Displacement graphic of mass $m 1$

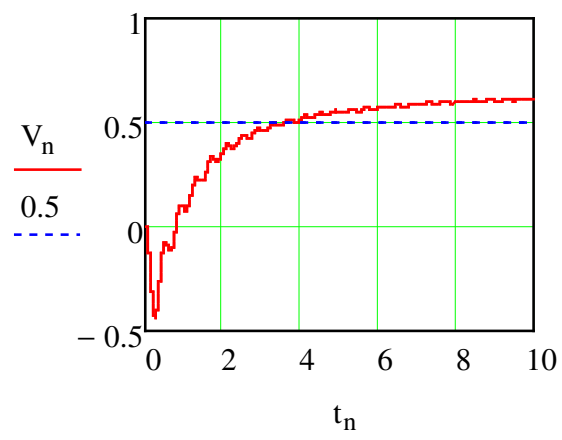

Fig. 8. Vibro driver velocity graphics in starting moment: middle velocity is more than $0.5 \mathrm{~m} \cdot \mathrm{s}^{-1}$ 


\section{Analysis of vibrodriver motion by Working Model}

Model (Fig. 4) was investigated, when inside programming control action (Fig. 5.) the actuator generates harmonica force. The position of body 1 and the velocity graphics of motion are shown in Fig. 9-12. The results of the movement in non-jump mode are approximately the same as those previously calculated by MathCAD (Fig. 6-8). The average speed on the inclined surface decreases (Fig. 12).

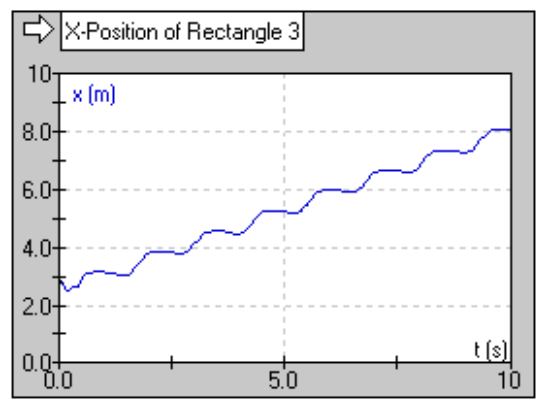

Fig. 9. Horizontal displacement graphics of mass $m 1$

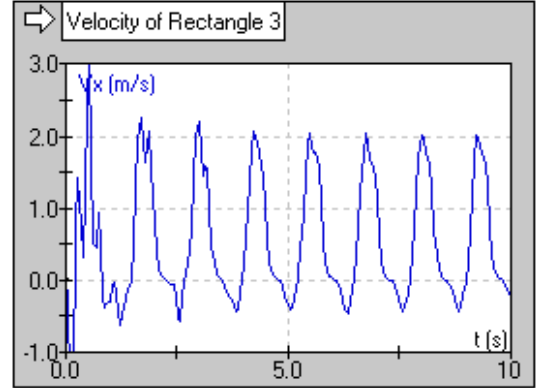

Fig. 10. Velocity $x$ - component graphics of mass $m 1$ : velocity is in limits -0.5 to 2 ; middle velocity is about $0.5 \mathrm{~m} \cdot \mathrm{s}^{-1}$

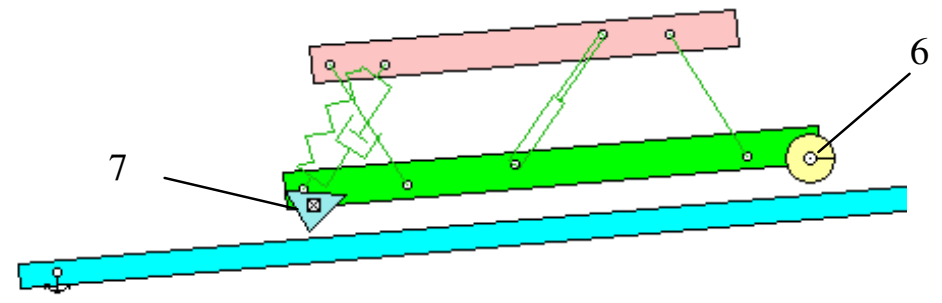

Fig. 11. Five degree of freedom (5 DOF) vibrodriver on a sloping surface:

6 - roller; 7-sharp element

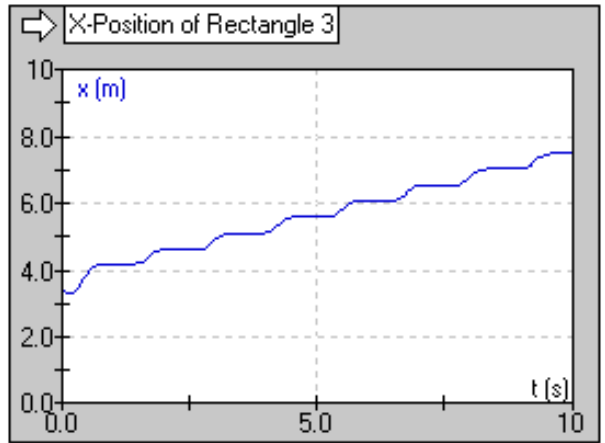

Fig. 12. Along slope surface displacement $x$ graphics of mass $m 1$

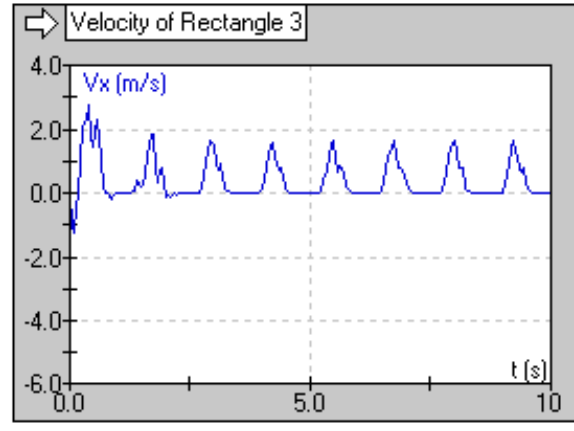

Fig. 13. Velocity $x$ - component graphics of mass $m 1$ : velocity is in limits -0.5 to 2 ; middle velocity is about $0.4 \mathrm{~m} \cdot \mathrm{s}^{-1}$

\section{Analysis of different vibro driver modifications}

Various degrees of freedom systems (4DOF - 9DOF) and drive types are described in Fig. 13-20. Comments on the type of excitation and the results obtained are given below each image. The conclusions are given at the end of the section. 


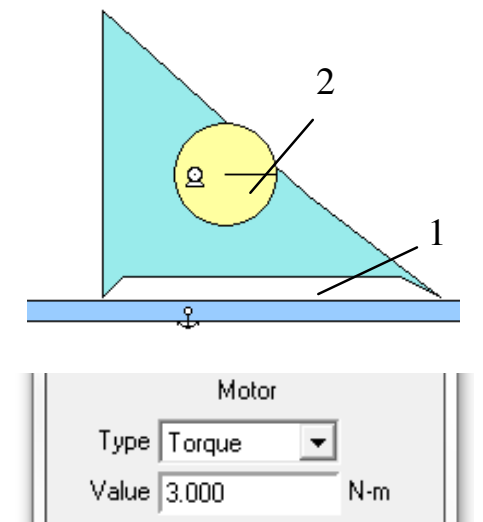

Fig. 14. Four degrees of freedom model:

1 - hull; 2 - disbalance of rotating actuator with given torque

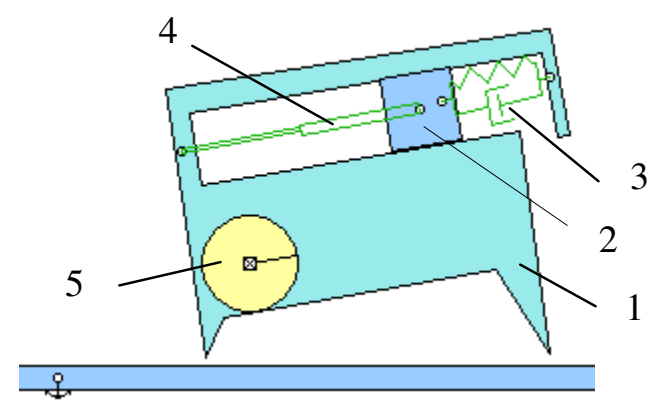

Fig. 16. Four degrees of freedom model (4DOF): 1 - hull; 2 - internal slider; 3 - spring and damper; $4-$ force $(20 \cdot \sin (5 t))$ actuator; 3 - balancing mass

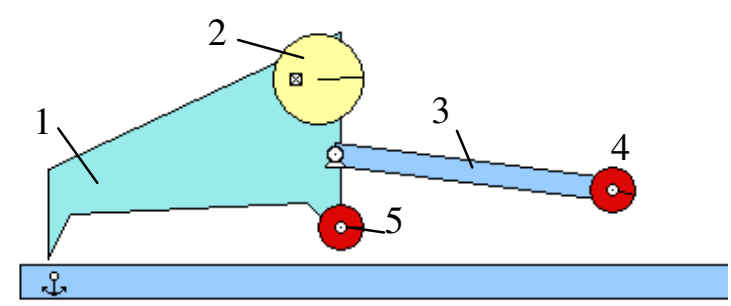

Fig. 18. Seven degrees of freedom model

(7DOF): 1 - hull; 2 - disbalance of rotating actuator with given torque $(25 \cdot \sin (5 t)) ; 3-\operatorname{rod}$; 4 - right roller; 5 - middle roller

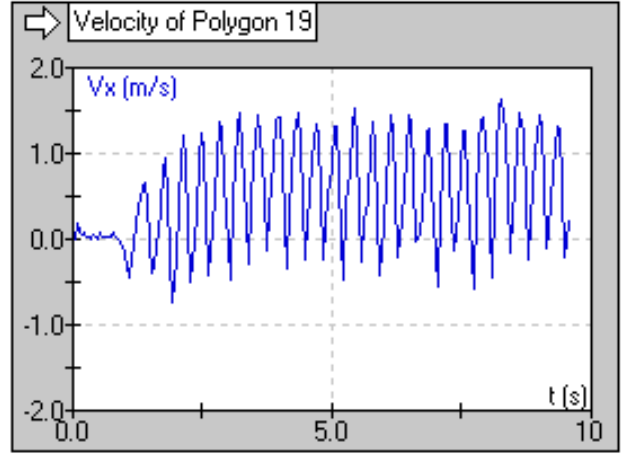

Fig. 15. Velocity $x$ - component graphics of mass $m$ 1: velocity is in limits $\mathbf{- 0 . 5}$ to 2 . Middle velocity is about $0.4 \mathrm{~m} \cdot \mathrm{s}^{-1}$

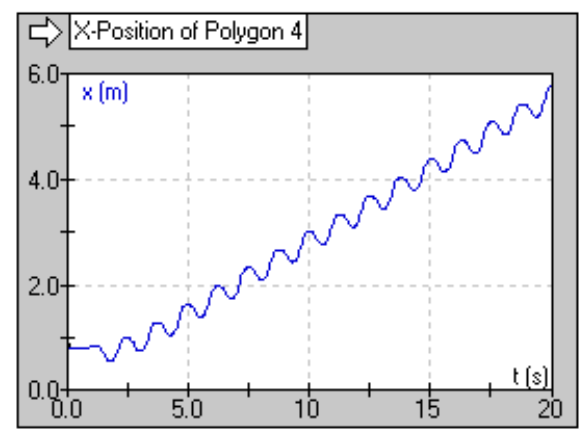

Fig. 17. Hull displacement: Middle velocity is about $0.5 \mathrm{~m} \cdot \mathrm{s}^{-1}$

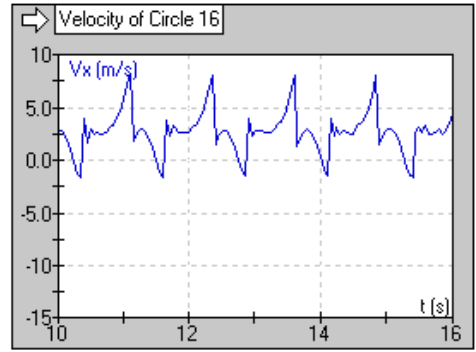

Fig. 19. Hull displacement: Middle velocity is about $2 \mathrm{~m} \cdot \mathrm{s}^{-1}$. Exists jumping mode

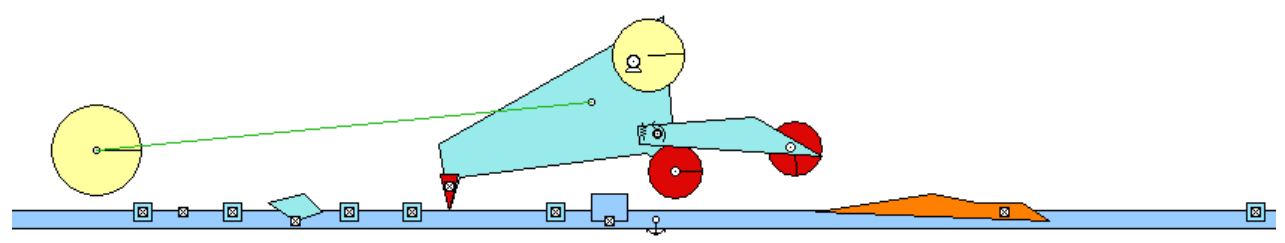

Fig. 20. Nine degrees of freedom model (9DOF): middle velocity is about $2 \mathbf{m} \cdot \mathrm{s}^{-1}$; jumping mode exists; it is possible to cross obstacles 


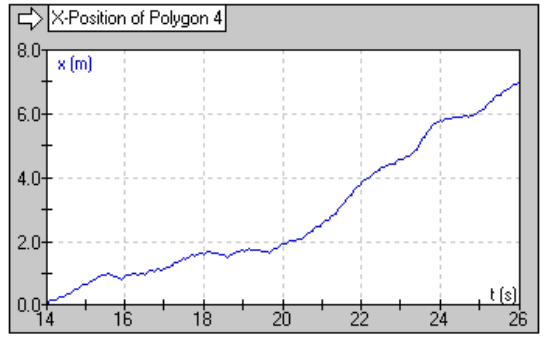

Fig. 21. Middle roller horizontal displacement

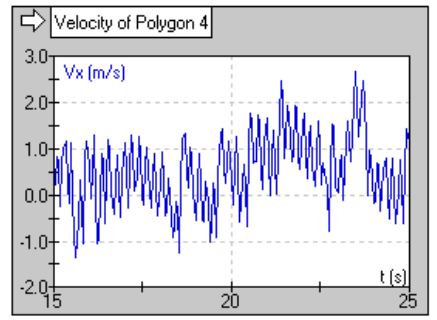

Fig. 22. Middle roller horizontal velocity

\section{Results and discussion}

1. Two possibilities for analyzing the motion of a robotic vibro engine are shown, i.e. using analytical formulas or modeling motion with discrete models of computer programs.

2. It has been found that discrete models must be used in the analysis of the motion of jumping robots with two (2DOF) and up to nine (9DOF) degrees of freedom, because the formulas of analytical methods are already included in these models.

3. However, analytical formulas also have their advantages, for example, in two- and three-degreeof-freedom models, it is possible to obtain analytical rules, following which the movement of a robot begins.

\section{Conclusions}

1. The methodology of vibration transport $2 \mathrm{DOF}$ motion calculation in analytical form is proposed using the law of classical mechanics, i.e. the mass center movement theorem for bulk material.

2. The method allows optimization of the technological process by selecting the excitation parameters.

3. It has been shown that in the very complex analysis of collision processes, the Working Model program was used effectively.

\section{References}

[1] Ragulskene V. L. Vibro-impact systems. Vilnius: Mintis, 1974. 320. p.

[2] Babitsky V.I. The theory of vibro-impact systems. Moscow, Nauka. 1978. 352. p. (In Russian).

[3] Kobrinsky A.A, Kobrinsky A. E. Two-dimensional vibration-shock systems: Dynamics and stability. Moscow. Nauka. 1981. 335. p. (In Russian).

[4] Ragulskis K. Vibrotechnika 50. Kaunas, Vibroengineering, 2013, 204 p. (in Lithuanian).

[5] Viba J. Optimization and synthesis of vibro impact machines. Riga. Zinatne. 1988. 252. p.(In Russian).

[6] Viba J., Polukoshko S. Simultaneous collisions of rigid bodies in more than one point. Proceedings of 4th International DAAAM Conference "Industrial Engineering - Innovation as Competitive Edge for SME “, April 2004, Tallinn, Estonia, pp.298-301.

[7] Linear vibrating separator. [online][11.02.2020] Available at: http://www.vibro-sieve.com/products/screening-equipment/linear-vibrating-screen.html

[8] Working Model 3D. Available at: https://working-model-3d.software.informer.com/3D 